\title{
Enrofloxacin decreases IL-6 and TNF- $\alpha$ production by lipopolysaccharide-stimulated porcine peripheral blood mononuclear cells
}

\author{
Małgorzata Pomorska-Mól, Ewelina Czyżewska-Dors, \\ Krzysztof Kwit, Zygmunt Pejsak \\ Department of Swine Diseases, \\ National Veterinary Research Institute, 24-100 Pulawy, Poland \\ mpomorska@piwet.pulawy.pl
}

Received: January 27, 2016 Accepted: May 10, 2016

\begin{abstract}
Introduction: The aim of the study was to explore the effect of enrofloxacin on production of selected cytokines by porcine peripheral blood mononuclear cells (PBMCs). Material and Methods: Twenty pigs (10 control and 10 experimental) were used in this research. Pigs from experimental group received enrofloxacin at therapeutic doses. Blood samples were collected before, during, and after treatment with enrofloxacin. PBMCs were incubated with or without lipopolysaccharide (LPS). Production of IL-6, IL-10, INF- $\gamma$, and TNF- $\alpha$ were determined by ELISA. Results: Administration of enrofloxacin to healthy pigs for $5 \mathrm{~d}$ induced a transient reduction of the PBMCs response to LPS in terms of IL- 6 and TNF- $\alpha$ secretion. The concentration of IL- 6 returned to the day 0 level shortly after treatment, while TNF- $\alpha$ production remained reduced for $10 \mathrm{~d}$ after the treatment. The production of IL-10 was not affected by enrofloxacin. The level of IFN- $\gamma$ was below the detection limit of the tests. Conclusion: The results indicate that enrofloxacin administered in vivo in therapeutic doses has an immunomodulatory effect through its capacity to inhibit secretion of IL- 6 and TNF- $\alpha$ by porcine PBMC stimulated by LPS.
\end{abstract}

Keywords: swine, fluoroquinolone, immunomodulation, lipopolysaccharide, cytokines.

\section{Introduction}

A wide range of antibiotics have been found to have immunomodulatory properties in addition to their antimicrobial activity $(6,17)$. It has been demonstrated that some fluoroquinolones can modulate host immune and inflammatory responses and cytokine production (1, 7-9, 14, 21). Khan et al. (9) have found that trovafloxacin suppresses the production of IL-1, IL-6, IL-10, and TNF- $\alpha$ by lipopolisaccharide (LPS)stimulated monocytes. Ciprofloxacin and ofloxacin have been shown to reduce the production of TNF- $\alpha$ in LPSstimulated monocytes (14). Araujo et al. (1) have demonstrated that moxifloxacin inhibited LPSstimulated secretion of IL-1 $\alpha$, IL-6, IL-10, IL-12, and TNF- $\alpha$. The evidence of the role of cytokines in the severity of infections as well as in the immune response indicate the importance of defining the immunomodulatory activity of antibiotics used to treat infections $(15,18)$.
Enrofloxacin is an antimicrobial agent which belongs to fluoroquinolones (25). Because of its pharmacokinetic properties, broad spectrum of activity, and low toxicity, enrofloxacin is frequently used by veterinarians to treat infections in swine (25). It is recommended for treatment of gastrointestinal and respiratory tract infections.

There are data on the effects of enrofloxacin treatments on the immune response in animals $(8,20$, 25 ), but the influence of in vivo administration of enrofloxacin on the cytokine secretion profile has so far not been documented. However, the results of earlier studies revealed that ciprofloxacin, a pharmacologically active metabolite of enrofloxacin, has significant effects on the production of wide range of cytokines by various cells $(2,14,24)$.

The evidence that fluoroquinolones have the capacity to modulate the production of cytokines by immune cells prompted us to study the effects of enrofloxacin given in therapeutics doses on the ex vivo 
production of cytokines by porcine peripheral blood mononuclear cells (PBMCs).

\section{Material and Methods}

Animals. Twenty 8 - to 20 -week-old pigs, both sexes, originating from a herd with high health status, were used in the study. Only pigs which had not received any antibiotics before the experiment were involved in the trial. The animals were randomly divided into two groups: control $(\mathrm{C} ; \mathrm{n}=10)$ and experimental $(\mathrm{E} ; \mathrm{n}=10)$. During the study, the pigs were housed in isolated units, one for each group, and were subjected to a $7 \mathrm{~d}$ acclimation period. Food and water were offered ad libitum. Animal use and handling protocols were approved by the Local Ethical Commission (University of Life Sciences in Lublin, Poland).

Drug. The commercial product containing enrofloxacin was used (Enrobioflox 5\% Injectio, $50 \mathrm{mg} / \mathrm{ml}$, Vetoquinol Biowet, Poland).

Experimental design. From day 0 to day 4, pigs from group $\mathrm{E}$ received enrofloxacin intramuscularly at the recommended therapeutic dose $(1 \mathrm{~mL} / 10 \mathrm{~kg}$ b.w. per day). Pigs from group $\mathrm{C}$ were intramuscularly administered the appropriate volume of normal saline (1 mL/10 kg b.w. per day).

Blood samples were collected from the vena jugularis or vena cava cranialis to vacuum tubes, containing EDTA-K $\mathrm{K}_{3}$ as an anticoagulant (Medlab, Poland) at days 0 (before antibiotic administration), 2, 4 (during antibiotic therapy), and 6, 9, 14, 21, 35, 49, and 63 (after treatment with enrofloxacin).

Measurements. PBMCs were isolated from blood samples by centrifugation onto Histopaque 1.077 (Sigma, USA). The cells were counted in Bürker chamber and their viability was determined with trypan blue (Stem Cell Technologies, UK) staining. Cytokine (IL-6, IL-10, IFN- $\gamma$, and TNF- $\alpha$ ) production by PBMCs was determined using cells cultured in plastic vials at a density of $1 \times 10^{6}$ viable cells $/ \mathrm{mL}$ medium (RPMI 1640 containing $10 \%$ foetal bovine serum, $2 \mathrm{mM}$ L-glutamine, and $1 \%$ of antibiotic-antimycotic solution) at $37^{\circ} \mathrm{C}$ and in a humidified $5 \%$ carbon dioxide atmosphere in the presence of $1 \mu \mathrm{g} / \mathrm{mL}$ lipopolysaccharide (LPS) (Escherichia coli 0111:B4; Sigma, USA). Simultaneously, unstimulated PBMCs were cultured under the same conditions. All samples were analysed in quadruplicate. After $24 \mathrm{~h}$, the culture media were harvested and centrifuged at $400 \times \mathrm{x}$ for $3 \mathrm{~min}$, and the supernatants were collected and stored at $-80^{\circ} \mathrm{C}$ until analysis.

To determine the concentrations of investigated cytokines in the culture supernatants, the ELISA kits specific for porcine IL- 6 , IL-10, IFN- $\gamma$, and TNF- $\alpha$ were used following the manufacturer's instructions (Invitrogen Corporation, USA; Abcam, UK). The absorbance was recorded at $450 \mathrm{~nm}$ using an ELISA plate reader (Multiskan RC, Labsystems, Finland).
Quantification was performed on the basis of a standard curve derived by dilution of the cytokine standards included in the kits. Concentrations of each cytokine were calculated from calibration curve using the FindGraph software. The detection limits of the kits were $2 \mathrm{pg} / \mathrm{mL}$ (IL-6), $2 \mathrm{pg} / \mathrm{mL}$ (IL-10), $2 \mathrm{pg} / \mathrm{mL}$ (IFN- $\gamma$ ), and $3 \mathrm{pg} / \mathrm{mL}$ (TNF- $\alpha$ ).

Statistical analysis. Data were expressed as the mean and standard deviation (SD). The data from both groups were subjected to the W. Shapiro-Wilk's test of normality and the Levene's test of equal variances. Because of non-normal distribution of the data, comparisons between the groups at each time point were assessed using the U Mann-Whitney test. The significance of changes in continuous data was evaluated by nonparametric Friedman test. Differences with $\alpha<0.05$ were considered as significant. Calculations were performed with the use of Statistica 8.0 software (Statsoft, Poland).

\section{Results}

Treatment of PBMCs with LPS resulted in a significant $(\mathrm{P}<0.05)$ increase in accumulation of IL-6, IL-10, and TNF- $\alpha$ in the cell culture supernatants when compared with unstimulated cells. The concentration of IFN- $\gamma$ after LPS stimulation was always below the detection limit in both groups of pigs (C and $\mathrm{E})$. No detectable levels of these cytokines were found in unstimulated cultures either.

There were no differences between the groups regarding IL-6 concentrations in supernatants at any time points $(\mathrm{P}<0.05)$ after mock-stimulation (Fig.1). The maximal concentration of IL-6 did not exceed $34 \mathrm{pg} / \mathrm{mL}$. In contrast, significant differences were found after LPS stimulation between IL-6 production by PBMCs isolated from pigs treated and not treated with enrofloxacin $(\mathrm{P}<0.05)$. The concentrations of IL- 6 in supernatants from LPS-stimulated cultures were significantly lower during and just after treatment with enrofloxacin (from day 2 to 6) in comparison with control animals $(\mathrm{P}<0.05)$. At days 2,4 , and 6 , IL-6 production was significantly decreased in enrofloxacin-treated pigs compared with day 0 level $(\mathrm{P}<0.05)$. Starting from day 9, no significant differences were found in IL-6 production between both groups of animals $(\mathrm{P}>0.05)$.

Lipopolysaccharide treatment of PBMCs isolated from control and enrofloxacin-treated animals resulted in a significant $(\mathrm{P}<0.05)$ increase in accumulation of IL-10 in the cell culture supernatants when compared with non-stimulated cultures. Ex vivo secretion of IL-10 by LPS- or mock-stimulated PBMCs was not affected significantly $(\mathrm{P}>0.05)$ by in vivo treatment with enrofloxacin (Fig. 2.)

Fig. 3 shows that TNF- $\alpha$ production in supernatants from mock-stimulated PBMCs did not differ between groups $(\mathrm{P}>0.05)$ and was stable during the period of study $(\mathrm{P}>0.05)$. In contrast, TNF- $\alpha$ production by LPS- 
stimulated PBMCs was significantly $(\mathrm{P}<0.05)$ higher in control group from day 2 to 14 of the study in comparison to enrofloxacin-treated pigs. Inhibition of $e x$ vivo secretion of TNF- $\alpha$ by PBMCs isolated from pigs treated with enrofloxacin was observed during antibiotic therapy and up to $10 \mathrm{~d}$ after treatment. Starting from day 21, no significant differences in LPS-induced production of this cytokine were noted between both groups $(\mathrm{P}>0.05)$.

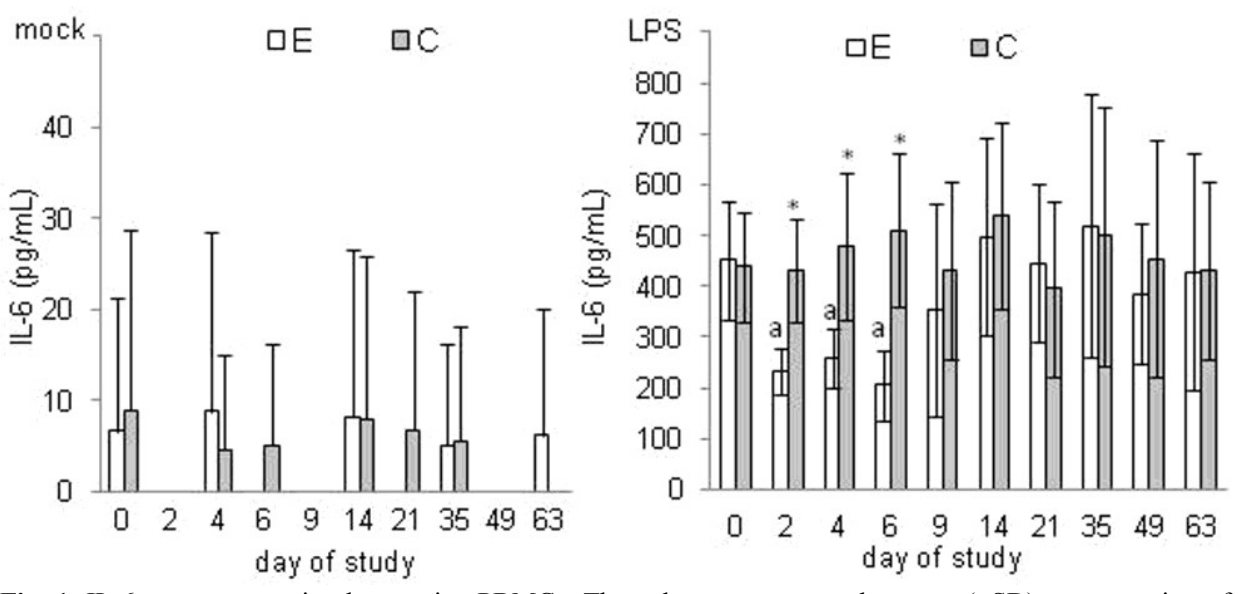

Fig. 1. IL-6 ex vivo secretion by porcine PBMCs. The columns represent the mean $( \pm \mathrm{SD})$ concentration of IL-6 in culture supernatant in unstimulated cultures (mock) and cultures stimulated with lipopolysaccharide (LPS). In the case of non-detectable level of this cytokine, the $0 \mathrm{pg} / \mathrm{mL}$ value was taken for calculation.

$\mathrm{E}$ - pigs treated with enrofloxacin at therapeutic doses for five consecutive days; $\mathrm{C}$ - control pigs

* - $\mathrm{P}<0.05$ as compared with group $\mathrm{E} ; \mathrm{a}-\mathrm{P}<0.05$ as compared with day 0 level
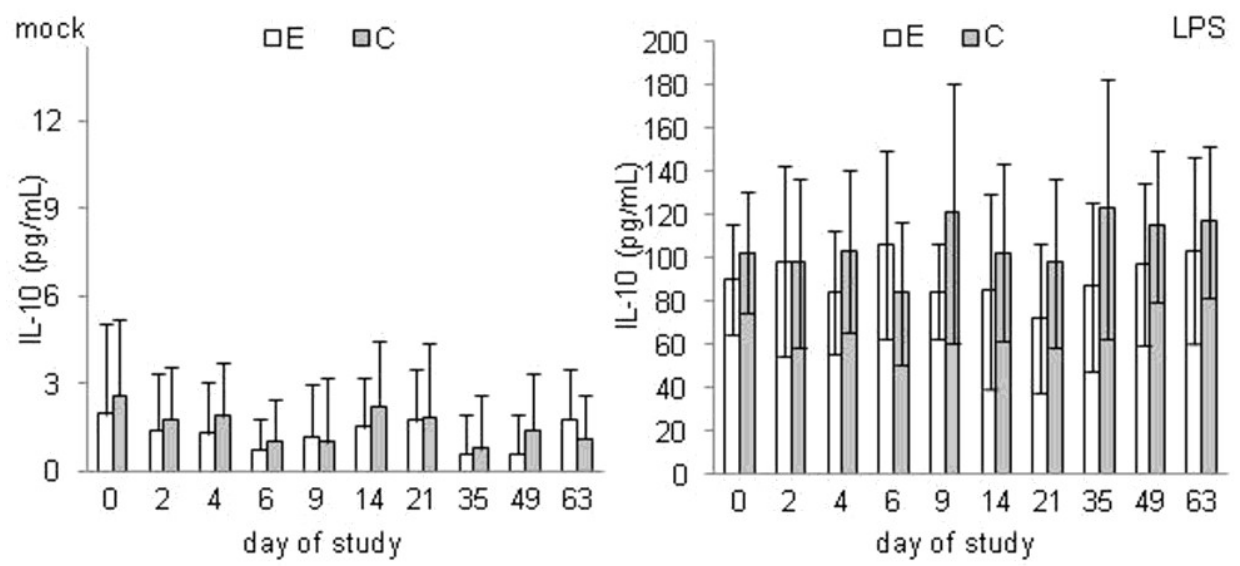

Fig. 2. IL-10 ex vivo secretion by porcine PBMCs. The columns represent the mean $( \pm \mathrm{SD})$ concentration of IL10 in culture supernatant in unstimulated cultures (mock) and cultures stimulated with lipopolysaccharide (LPS) $\mathrm{E}$ - pigs treated with enrofloxacin at therapeutic doses for five consecutive days; $\mathrm{C}$ - control pigs

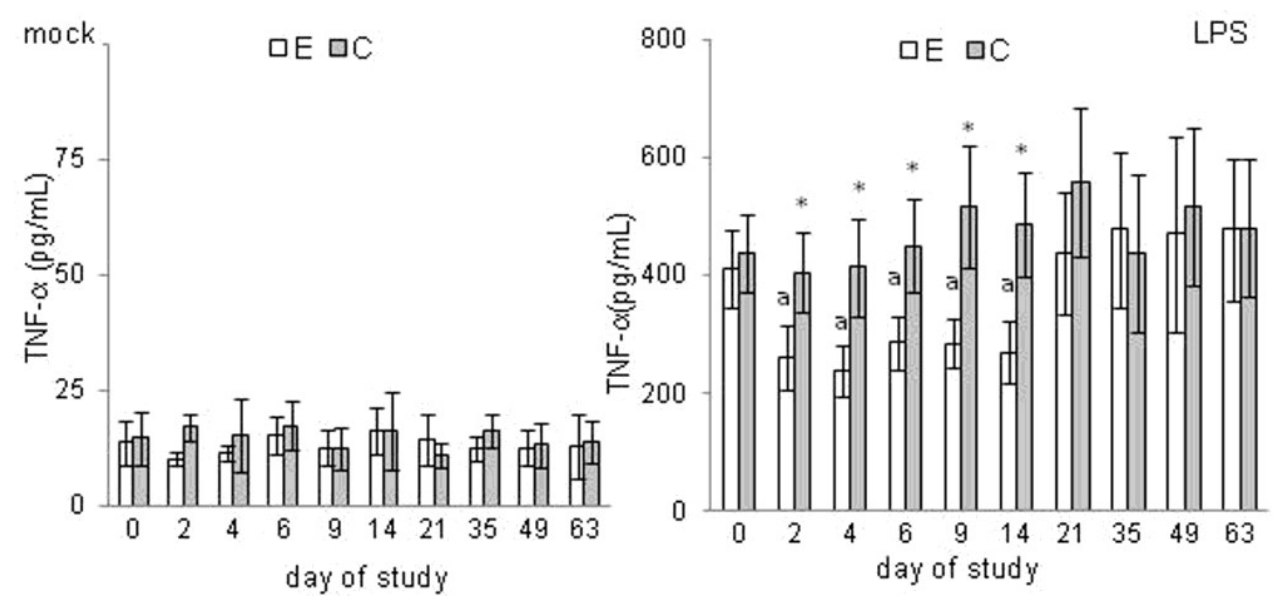

Fig. 3. TNF- $\alpha$ ex vivo secretion by porcine PBMCs. The columns represent the mean ( \pm SD) concentration of TNF- $\alpha$ in culture supernatant in unstimulated cultures (mock) and cultures stimulated with lipopolysaccharide (LPS)

$\mathrm{E}$ - pigs treated with enrofloxacin at therapeutic doses for five consecutive days; $\mathrm{C}$ - control pigs

* - $\mathrm{P}<0.05$ as compared with E group; a $-\mathrm{P}<0.05$ as compared with day 0 level 


\section{Discussion}

Intramuscular administration of enrofloxacin to healthy pigs for five consequtive days induced a transient reduction of ex vivo response of PBMCs to LPS in terms of IL-6 and TNF- $\alpha$ secretion. The concentration of IL- 6 returned to the day 0 level shortly after the end of treatment, while the TNF- $\alpha$ production remained reduced longer, up to $10 \mathrm{~d}$ after the end of treatment. The production of IL-10, an anti-inflamatory cytokine, was not affected by the enrofloxacin treatment. The supression of IL- 6 and TNF- $\alpha$ secretion occurred in all animals treated with enrofloxacin. At the same time the spontaneus production of these cytokines (unstimulated cells) was not modified. The results described above revealed that enrofloxacin can reduce production of IL-6 and TNF- $\alpha$ porcine immunocompetent cells, which suggests that, in addition to its antimicrobial effects, enrofloxacin may limit the immune and inflamatory reaction occurring in the course of certain infections.

The role of cytokines investigated in the present study is significant in the immune response to various pathogens. IL- 6 is a cytokine of innate immunity and regulates inflammation and transition from innate to adaptive immune responses. It is also important in haematopoiesis and acute phase response. The early secretion of this cytokine may constitute an important line of defence against pathogens (11). IL-10 is a cytokine secreted by various immune cells. It has pleiotropic effects in immunoregulation and inflammation. IL-10 can down-regulate major histocompatibility complex class II expression, synthesis of other cytokines, and its own production, and is able to inhibit the functions of the natural killer (NK) cells, lymphocytes and monocytes (13, 15). TNF- $\alpha$, produced mainly by activated macrophages, CD4+ lymphocytes and NK cells, can stimulate neutrophil activation, synthesis of acute phase proteins, and apoptosis of cells. It is also involved in T-cell activation. Bacterial endotoxins, a range of molecules of pathogens, and other cytokines are believed to be principal stimuli for TNF- $\alpha$ release from cells (3). The reduction of the TNF- $\alpha$ production may affect neutrophil activation and modulate the immune response against pathogens.

Previously, other fluoroquinolones have been evaluated for their effect on the cytokine production in vitro or ex vivo $(1,9,19,22)$. Our results regarding the significant reduction in LPS-induced IL- 6 and TNF- $\alpha$ secretion are in agrrement with previous findings from various in vitro studies $(1,9,19,22)$. Khan et al. (9) have found that trovafloxacin, an antimicrobial drug belonging to fluoroquinolones, suppressed the secretion of various cytokines, including IL-6, IL-10, and TNF- $\alpha$, by monocytes cultivated in vitro after LPS stimulation. The data presented by Tsivkovskii et al. (22) have demonstrated that levofloxacin also reduces LPSinduced IL-6 levels in cultured human airway epithelial cells. In contrast, Bailly et al. (2) have found that ciprofloxacin treatment in vivo in humans increases the ex vivo capacity of LPS-stimulated human monocytes to produce IL- 6 and TNF- $\alpha$. However, in the same study the extracellular production of IL- 6 was reduced after treatment with ciprofloxacin (2).

Our results revealed that stimulation of porcine PMBCs with LPS resulted in a significant increase in the production of most of the investigated cytokines in supernatants of the cultured PBMCs isolated from both enrofloxacin treated and non-treated pigs, when compared with unstimulated cells. Similar results were reported by Araujo et al. (1) who investigated the in vitro influence of moxifloxacin on secretion of cytokines by human monocytes stimulated with LPS. They have also found that moxifloxacin, similarly as enrofloxacin, significantly inhibited secretion of TNF- $\alpha$.

In the present study only the level of IFN- $\gamma$ was below detection limit in supernatants of the LPSstimulated as well as unstimulated PBMCs cultured in vivo. The lack of detectable levels of IFN- $\gamma$ in the culture supernatants after LPS-stimulation is not surprising since this cytokine is produced mostly by activated $\mathrm{T}$ lymphocytes and NK cells $(5,23)$. LPS stimulates mainly, but not solely, B lymphocytes, monocytes, and macrophages, the cells that express the LPS receptor (23). There is also evidence that activated human, but not murine, CD8 $+\mathrm{T}$ cells can secrete high concentrations of IFN- $\gamma$ in response to LPS (10). It is important to note that LPS sensitivity differs considerably among species (23). To date there is no information regarding reactivity of porcine $\mathrm{T}$ cells upon LPS stimulation. However, the results of the present study suggest that porcine $\mathrm{T}$ lymphocytes, similarly to murine T lymphocytes, do not respond with significant IFN- $\gamma$ production after LPS stimulation.

In conclusion, our results indicate that enrofloxacin has an immunomodulatory effect through its capacity to inhibit ex vivo secretion of IL-6 and TNF- $\alpha$ by porcine PBMC after LPS stimulation. Because of the important role of these cytokines in the host immune response, this effect may influence the course of infection in a manner that is independent of the drug's antimicrobial activity. The inhibiting effects of enrofloxacin on the production of IL- 6 and TNF- $\alpha$ in response to LPS may be beneficial through modulation of the inflammatory response to the invading pathogens, especially during bacteraemia, which may lead to massive production of cytokines $(12,15,19)$. Overproduction of proinflammatory cytokines (including IL-6 and TNF- $\alpha$ ) may result in a range of pathological conditions, including septic shock (4). It is worth noting that the beneficial effects of in vivo treatment with ciprofloxacin, an active metabolite of enrofloxacin, were previously reported by Purswani et al. (19), who found that ciprofloxacin can prevent endotoxin-mediated death in mice and alter early host cytokine responses. 
Conflict of Interests Statement: The authors declare that there is no conflict of interests regarding the publication of this article.

Financial Disclosure Statement: This work was supported by the National Science Centre (DEC2012/05/B/NZ7/03114).

Animal Rights Statement: The experiment was approved by Local Ethics Commission (University of Life Sciences in Lublin, Poland).

\section{References}

1. Araujo F.G., Slifer T.L., Remington J.S.: Effect of moxifloxacin on secretion of cytokines by human monocytes stimulated with lipopolysaccharide. Clin Microbiol Infect 2002, 8, 26-30.

2. Bailly S., Fay M., Ferrua B., Gougerot-Pocidalo M.A.: Ciprofloxacin treatment in vivo increases the ex vivo capacity of lipopolysaccharide-stimulated human monocytes to produce IL-1, IL-6 and tumour necrosis factor-alpha. Clin Exp Immunol 1991, 85, 331-334.

3. Bazzoni F., Beutler B.: The tumor necrosis factor ligand and receptor families. New Engl J Med 1996, 334, 1717-1725.

4. Chirigos N.A., De Simone C.: Immunoregulatory biological response modifiers: effect of cytokines on septic shock. Mediators Inflam 1993, 2, 5-10.

5. Choi I.S., Collisson E.W., Maheswaran S.K., Yoo H.S.: Evaluation of cytokine gene expression in porcine spleen cells, peripheral blood mononuclear cells, and alveolar macrophages by competitive RT-PCR. FEMS Immunol Med Microbiol 2002, 34, 119-126.

6. Dalhoff A.: Immunomodulatory activities of fluoroquinolones. Infection 2005, 33, 255-270.

7. Gattoni A., Parlato A., Vangieri B., Bresciani M., Derna R.: Interferon-gamma: biologic functions and $\mathrm{HCV}$ therapy (type I/II). Clin Therap 2006, 157, 377-386.

8. Khalifeh M.S., Amawi M.M., Abu-Basha E.A., Bani Yonis I.: Assessment of humoral and cellular-mediated immune response in chickens treated with tilmicosin, florfenicol or enrofloxacin at the time of Newcastle disease vaccination. Poultry Sci 2009, 88, 2118-2124.

9. Khan A.A., Slifer T.R., Remington J.S.: Effect of trovafloxacin on production of cytokines by human monocytes. Antimicrob Agent Chemother 1998, 42, 1713-1717.

10. Koma-Komai M., Gilchrist D.S., Xu D.: Direct recognition of LPS by human but not murine CD8+ T cells via TLR4 complex. Eur J Immunol 2009, 39, 1564-1572.

11. Lauder S.N., Jones E., Smart K., Bloom A., Williams A.S., Hindle J.P., Ondondo B., Taylor P.R., Clement M., Fielding C., Godkin A.J., Jones S.A., Gallimore A.M.: Interleukin-6 limits influenza-induced inflammation and protects against fatal lung pathology. Eur J Immunol 2013, 43, 2613-2625.

12. Marsh C.B., Wewers M.D.: The pathogenesis of sepsis. Factors that modulate the response to gram-negative bacterial infection. Clin Chest Med 1996, 7, 183-197.

13. Moore K.W., de Waal Malefyt R., Coffman R.L., O'Garra A.: Interleukin-10 and the interleukin-10 receptor. Ann Rev Immunol 2001, 19, 683-765.

14. Morikawa K., Watabe H., Araake M., Morikawa S.: Modulatory effect of antibiotics on cytokine production by human monocytes in vitro. Antimicrob Agent Chemother 1996, 40, 1366-1370.

15. Opal S.M., Esmon C.T.: Bench-to-bedside review: functional relationships between coagulation and the innate immune response and their respective roles in the pathogenesis of sepsis. Critical Care 2003, 7, 23-38.

16. Parveen Y., Calder P.C.: Cytokine production by human peripheral blood mononuclear cells: differential sensitivity to glutamine availability. Cytokine 1998, 10, 790-794.

17. Pomorska-Mól M., Kwit K., Markowska-Daniel I., Pejsak Z.: The effect of doxycycline treatment on the postvaccinal immune response in pigs. Toxicol Appl Pharmacol 2014, 278, 31-38.

18. Pomorska-Mól M., Markowska-Daniel I., Kwit K., Czyżewska E., Dors A., Rachubik J., Pejsak Z.: Immune and inflammatory response in pigs during acute influenza caused by H1N1 swine influenza virus. Arch Virol 2014, 159, 2605-2614.

19. Purswani M.U., Eckert S.J., Arora H.K., Moel G.J.: Effect of ciprofloxacin on lethal and sublethal challenge with endotoxin and on early cytokine response in a murine in vivo model. J Antimicrob Chemother 2002, 50, 51-58.

20. Schoevers E.J., van Leengoed L.A.M.G., Verheijden J.H.M., Niewold T.A.: Effects of enrofloxacin on porcine phagocytic function. Antimicrob Agent Chemother 1999, 43, 2138-2143.

21. Shalit I., Halperin D., Haite D., Levitov A., Romano J., Osherov N., Fabian I.: Anti-inflammatory effects of moxifloxacin on IL-8, IL-1beta and TNF-alpha secretion and NFkappaB and MAP-kinase activation in human monocytes stimulated with Aspergillus fumigatus. J Antimicrob Chemother 2006, 57, 230-235.

22. Tsivkovskii R., Sabet M., Tarazi Z., Griffith D.C., Lomovskaya O., Dudley M.N.: Levofloxacin reduces inflammatory cytokine levels in human bronchial epithelia cells: implications for aerosol MP-376 (levofloxacin solution for inhalation) treatment of chronic pulmonary infections. FEMS Immunol Med Microbiol 2011, 61, 141-146.

23. Vaure C., Liu Y.: A comparative review of toll-like receptor 4 expression and functionality in different animal species. Front Immunol 2014, 5, 316.

24. Williams A.C., Galley H.F., Watt A.M., Webster N.R.: Differential effects of three antibiotics on $\mathrm{T}$ helper cell cytokine expression. J Antimicrob Chemother 2005, 56, 502-506.

25. Ziółkowski H., Jaroszewski J.J., Maślanka T., Grabowski T., Katolik K., Pawęska J., Siemianowska M., Jasiecka A., Markiewicz W., Spodniewska A.: Influence of oral coadministration of a preparation containing calcium and magnesium and food on enrofloxacin pharmacokinetics. Res Vet Sci 2014, 7, 99-104. 